\title{
Analyzing the Role of Receptor Internalization in the Regulation of Melanin-Concentrating Hormone Signaling
}

\author{
Jay I. Moden, Katrina Haude, Robert Carroll, Andrew Goodspeed, and Laurie B. Cook \\ Department of Biology, 217 Lennon Hall, The College at Brockport, State University of New York, 350 New Campus Drive, \\ Brockport, NY 14420, USA
}

Correspondence should be addressed to Laurie B. Cook; lcook@brockport.edu

Received 11 July 2013; Accepted 10 October 2013

Academic Editor: Stacia A. Sower

Copyright (c) 2013 Jay I. Moden et al. This is an open access article distributed under the Creative Commons Attribution License, which permits unrestricted use, distribution, and reproduction in any medium, provided the original work is properly cited.

\begin{abstract}
The regulation of appetite is complex, though our understanding of the process is improving. The potential role for the melaninconcentrating hormone $(\mathrm{MCH})$ signaling pathway in the treatment of obesity is being explored by many. It was hypothesized that internalization of $\mathrm{MCH}$ receptors would act to potently desensitize cells to $\mathrm{MCH}$. Despite potent desensitization of ERK signaling by $\mathrm{MCH}$ in $\mathrm{BHK}-570$ cells, we were unable to observe $\mathrm{MCH}$-mediated internalization of $\mathrm{MCH}$ receptor 1 (MCHR1) by fluorescence microscopy. A more quantitative approach using a cell-based ELISA indicated only 15\% of receptors internalized, which is much lower than that reported in the literature. When $\beta$-arrestins were overexpressed in our system, removal of receptors from the cell surface was facilitated and signaling to a leptin promoter was diminished, suggesting that internalization of MCHR1 is sensitive to cellular $\beta$-arrestin levels. A dominant-negative GRK construct completely inhibited loss of receptors from the cell surface in response to $\mathrm{MCH}$, suggesting that the internalization observed is phosphorylation-dependent. Since desensitization of $\mathrm{MCH}$-mediated ERK signaling did not correlate with significant loss of MCHR1 from the cell surface, we hypothesize that in this model system regulation of $\mathrm{MCH}$ signaling may be the result of segregation of receptors from signaling components at the plasma membrane.
\end{abstract}

\section{Introduction}

Obesity results when caloric intake exceeds metabolic needs over an extended period of time. The condition predicates heart disease and diabetes-two pathologies that diminish the quality of life and increase risk of premature death. While appetite control is incredibly complex, we do know that a hormone discovered in teleost fish, melanin-concentrating hormone $(\mathrm{MCH})$, plays a role in regulating feeding behavior in higher-order mammals [1-5]. MCH acts via a G proteincoupled receptor (GPCR) at the plasma membrane of many cell types including neurons to stimulate appetite and adipocytes to stimulate the synthesis and secretion of leptin. Studies with MCHR1 knockout mice have shown similar physiological adaptations including an increase in resistance to diet-induced obesity and hyperphagia compared to wildtype mice fed a similar diet [6]. Recently, loss of MCHR1 localization to primary cilia has been linked to the severe obesity seen with Bardet-Biedl syndrome [7], suggesting that regulation of $\mathrm{MCH}$ signaling plays an important role in energy homeostasis and that this pathway could become a pharmacological target to curb appetite. The usefulness of an MCHR1 antagonist in treating human obesity is being explored by others; an Early Phase I Clinical Trial has seen promising results [8].

The dramatic phenotype observed in loss of function studies suggests that irregular $\mathrm{MCH}$ signaling patterns have the potential to cause disease. Unless appetite signaling is properly desensitized following food consumption, hunger may continue. The desensitization of MCHR1 was first described by Lembo et al., who observed internalization of MCHR1 by fluorescence microscopy following agonist treatment [9]. This was supported by evidence that MCHR1 internalization occurs via the canonical $\beta$-arrestin and clathrinmediated pathway $[10,11]$. Flag-tagged MCHR1 internalized in HEK293T cells following $30 \mathrm{~min} \mathrm{MCH}$ treatment to only $44.2 \%$ of initial surface receptor levels [11]. Further studies suggested that $\beta$-arrestin 2 is preferentially recruited 
to MCHR1; however, they were performed in the presence of overexpressed GRK2, a kinase that increases the affinity of GPCRs for arrestins [10].

The goal of this study was to further characterize the agonist-mediated desensitization of MCHR1. We unexpectedly discovered early on that despite rapid and extensive desensitization of ERK activation in response to $\mathrm{MCH}$, agonist-mediated internalization of MCHR1 was very poor in our model, which is contrary to what others have reported $[10,11]$. Although we were able to significantly improve receptor internalization by overexpression of $\beta$-arrestins or GRK2, this study suggests that there is an unexplored desensitization mechanism that occurs at the plasma membrane in the absence of receptor internalization.

\section{Materials and Methods}

2.1. Tissue Culture. BHK-570 cells (ATCC) were cultured as a tissue monolayer using DMEM media (CellGro) containing $10 \%$ fetal bovine serum (Atlanta Biological) without antibiotics. Cells were fed every four days and passaged when they were confluent. Culture conditions were set at $37^{\circ} \mathrm{C}, 5 \% \mathrm{CO}_{2}$, and $80 \%$ humidity.

2.2. Plasmids. Plasmid DNA encoding MCHR1 in pcDNA3 was purchased from the Missouri S\&T cDNA Resource Center. pcDNA3.1+ plasmid encoding MCHR1-VSVg was obtained from Dr. G. Milligan at the University of Glasgow. Dr. J. Benovic at Thomas Jefferson University generously provided plasmids encoding $\beta$-arrestin 1 and $\beta$-arrestin 2 in pEGFP-N as well as GRK2 and GRK2 K220R in pRK5. The leptin promoter-driven luciferase reporter plasmid $\mathrm{p}(-762) \mathrm{ob}$ luc was a gift from Dr. O. Gavrilova at the NIH.

2.3. Transfection. Cell transfections were performed on cells plated for at least $24 \mathrm{~h}$ with LipoD293 reagent following the recommended protocol from SignaGen. Media were changed 5 hours after transfection and experiments were run approximately 48 hours after transfection.

2.4. ERK Activation Assay. BHK-570 cells in $35 \mathrm{~mm}$ dishes were transfected with $1 \mu \mathrm{g}$ MCHR1 in pcDNA3 or empty plasmid on day 1 . On day 2, cells were serum starved in DMEM for 18-24 hours. On the third day, baseline ERK activation was obtained by treating cells for up to $30 \mathrm{~min}$ with $1 \mu \mathrm{M} \mathrm{MCH}$ (rat, American Peptide) prior to harvesting in 2X Laemmli sample buffer. Desensitization assays were performed by treating cells with a desensitizing dose of $\mathrm{MCH}$ for $15 \mathrm{~min}$ followed by rinsing with and incubation of cells in DMEM in the incubator for $30 \mathrm{~min}$ (called washout). To activate the pathway again, cells were treated with a second dose of $\mathrm{MCH}$ for up to $30 \mathrm{~min}$ prior to harvesting in $2 \mathrm{X}$ Laemmli sample buffer. Lysates were boiled for $5 \mathrm{~min}$ and spun at top speed in a microcentrifuge prior to SDS-PAGE. Proteins were transferred to nitrocellulose and western blots performed with antibodies to both phosphorylated and total ERK (Cell Signaling). Bands were exposed using Western Lightning Enhanced Chemiluminescence kit (Perkin Elmer) on Kodak film.

2.5. Fixed Cell Fluorescence Microscopy. Coverslips seeded with BHK-570 cells were transfected with MCHR1-VSVg \pm GFP-tagged $\beta$-arrestin 1 or 2 . Cells were then incubated for approximately $24 \mathrm{~h}$ and then treated with $\pm 1 \mu \mathrm{M} \mathrm{MCH}$ (American Peptide) for up to $30 \mathrm{~min}$. The coverslips were then rinsed twice with ice-cold PBS before fixing with $4 \%$ paraformaldehyde in PBS for $10 \mathrm{~min}$ at room temperature. After 3 more washes with PBS, they were transferred to a humidification chamber for incubation in blocking buffer (PBS, 5\% goat serum, $0.1 \%$ Triton X-100) for at least $20 \mathrm{~min}$. They were then incubated for at least $1 \mathrm{~h}$ with rabbit antiVSVg polyclonal antibody (Sigma) at 1:50 in blocking buffer. Following 3 PBS washes, DAPI (Roche) was added at 1:500 and Alexa Fluor 546 goat anti-rabbit secondary antibody (Invitrogen) at 1:250 in blocking buffer for $1 \mathrm{~h}$. After 3 more PBS washes, coverslips were mounted on glass slides with Prolong Gold (Invitrogen). Fluorescence micrographs were obtained with a Zeiss Axioskop fluorescence microscope with a Zeiss Axiocam camera and Zeiss Axiovision software. Image cropping was performed in Adobe Photoshop and figures assembled in MS PowerPoint. For Figure 4, overlay composite color images were processed in NIH ImageJ to extract individual colors.

2.6. Cell-Based ELISA. Twenty-four well plates were seeded with BHK-570 cells and transfected with MCHR1-VSVg \pm GFP $\beta$-arrestin 1 or 2 or in a separate experiment GRK2 or K220R GRK2. Twenty-four hours after transfection, the cells were incubated in labeling buffer (DMEM, 5\% goat serum, 0.02 mM HEPES) with 1:1,000 mouse anti-VSVg (Sigma) for $2 \mathrm{~h}$. The cells were then washed twice in labeling buffer before being treated with $1 \mu \mathrm{M} \mathrm{MCH}$ for up to $30 \mathrm{~min}$. The cells were then washed once with ice cold PBS and fixed with $3 \%$ paraformaldehyde in PBS for $20 \mathrm{~min}$ at room temperature. Following 2, $5 \mathrm{~min}$ washes with PBS, cells were incubated with goat anti-mouse HRP-conjugated secondary antibody (Bio-Rad) in PBS with 5\% goat serum for 45 minutes. After another 3 PBS washes, $175 \mu \mathrm{L}$ of POD blue (Roche) was added to develop for $3 \mathrm{~min}$ on an orbital shaker. The reaction was neutralized with $175 \mu \mathrm{L}$ of $10 \%$ sulfuric acid. One hundred fifty microliters from each well were transferred to a 96-well plate and the absorbance was read at $450 \mathrm{~nm}$ using a Synergy 1 or ELx800 plate reader (BioTek).

2.7. Leptin Promoter Activation Assay. BHK-570 cells were triple transfected with either (1) MCHR1/p(-762)ob luc/GFP in pcDNA3, (2) MCHR1/p (-762)ob luc/GFP- $\beta$-arrestin 1 , or (3) $\mathrm{MCHR} 1 / \mathrm{p}(-762) \mathrm{ob}$ luc/GFP $\beta$-arrestin 2 . The cells were treated $48 \mathrm{~h}$ after transfection with $1 \mu \mathrm{M} \mathrm{MCH}$ for up to $6 \mathrm{~h}$ in DMEM and then lysed and luminol was added. Light production was measured over $15 \mathrm{sec}$ on a BioTek Synergy H1 plate reader. 


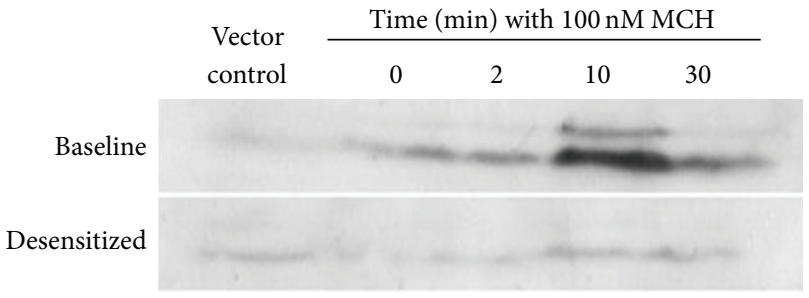

(a)

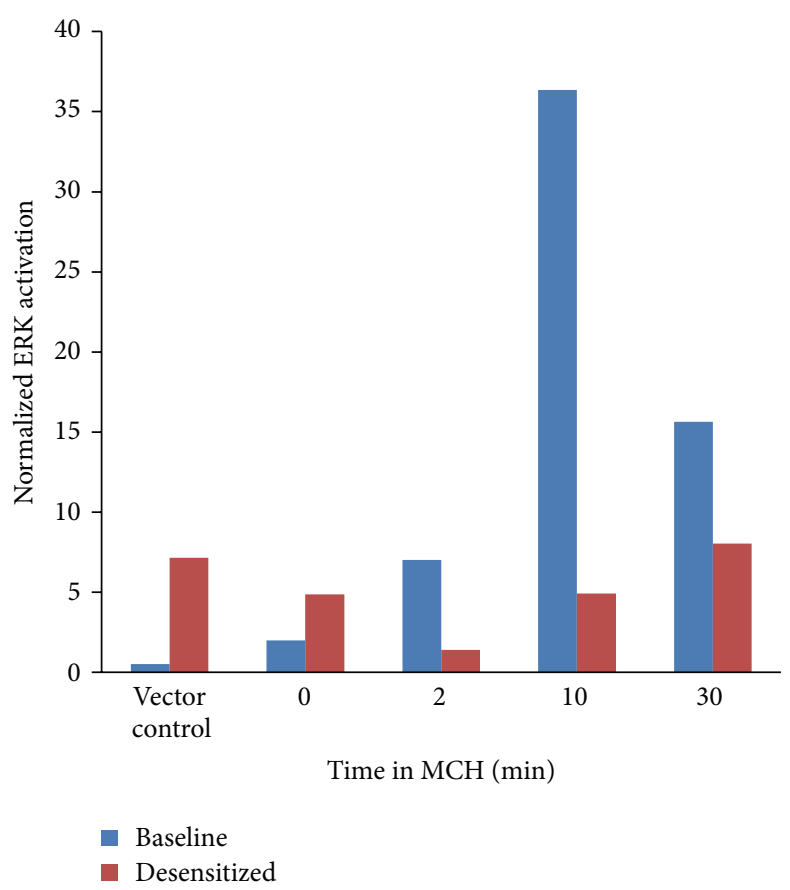

(b)

FIGURE 1: MCH-mediated ERK activation efficiently desensitizes. BHK-570 cells were transfected with plasmid DNA encoding MCHR1 an ERK activation assay performed on cells treated with $\mathrm{MCH}$ for up to $30 \mathrm{~min}$. A second set of dishes was treated for $\mathrm{MCH}$ for $15 \mathrm{~min}$ and then subjected to a $30 \mathrm{~min}$ washout prior to the $\mathrm{MCH}$ time course (2nd exposure). (a) Cells were harvested in $2 \mathrm{X}$ Laemmli sample buffer, run on SDS-PAGE and western blots were performed with primary antibodies to phosphorylated and total ERK. (b) Densitometry was performed using NIH Image J Software where ERK activation was normalized against total ERK. Experiment shown is representative of three total experiments.

2.8. Statistical Analyses. The averages of the data were reported \pm the standard error of the mean (SEM). Student's $t$ test was performed to determine statistical significance with data scoring equal to or greater than the 95th percentile considered significant.

\section{Results}

3.1. MCH-Mediated Desensitization of ERK Signaling. It was previously reported that $\mathrm{MCH}$ caused activation of the MAPK pathway and leptin promoter in 3T3-L1 adipocytes and that MCHR1 was subsequently downregulated in these cells [12]. To see if MCHR1 could signal to ERK in our model system, we transfected BHK-570 cells with MCHR1 and stimulated them with $\mathrm{MCH}$ for $0,2,10$, and $30 \mathrm{~min}$ prior to lysis. Western blots were performed using antibody directed towards phosphorylated and total ERK. We observed transient ERK activation in response to $\mathrm{MCH}$ that was not detectable in empty vector transfected control cells as seen in Figure 1(a) as baseline activation, which is quantitated in Figure 1(b) by normalizing activated ERK to total ERK (blots not shown). Within $10 \mathrm{~min}$ we measured a greater than 30-fold activation of ERK over baseline that disappeared by $30 \mathrm{~min}$. Surprisingly, when hormone was removed, cells were washed extensively and allowed to recover for up to 30 min and $\mathrm{MCH}$ did not regain its ability to activate ERK, indicating significant desensitization of the pathway (Figures $1(\mathrm{a})$ and $1(\mathrm{~b})$ ). We previously showed that an $\mathrm{N}$-terminally tagged VSVg-MCHR1 construct efficiently activates ERK in response to $\mathrm{MCH}$ binding [13], and preliminary desensitization experiments conducted with this construct in $\mathrm{CHO}-$ $\mathrm{K} 1$ cells had similar results to those in Figure 1. Therefore, we conclude that the presence of the VSVg tag does not interfere with receptor signaling (data not shown), and we took advantage of the sensitivity of this epitope to explore the role that receptor internalization plays in controlling the duration of $\mathrm{MCH}$-mediated ERK signaling.

3.2. A Small Percentage of MCHR1 Internalizes in Response to $\mathrm{MCH}$. The desensitization of MCHR1 was first described by Lembo et al., who observed internalization of MCHR1 through fluorescence microscopy following agonist treatment [9]. We hypothesized that internalization of MCHR1 could be responsible for the extensive desensitization of the ERK signaling pathway by MCH. Much to our surprise, we have been unable to observe any agonist-induced redistribution of MCHR1 using VSVg-tagged MCHR1 expressed in BHK-570 cells by fluorescence microscopy (Figure 2(a)). This was neither specific to the construct (MCHR1-eYFP did not internalize) nor was it specific to BHK-570 cells (both CHO-K1 cells and 3T3-L1 cells failed to internalize MCHR1) (data not shown). We thought that perhaps our method 


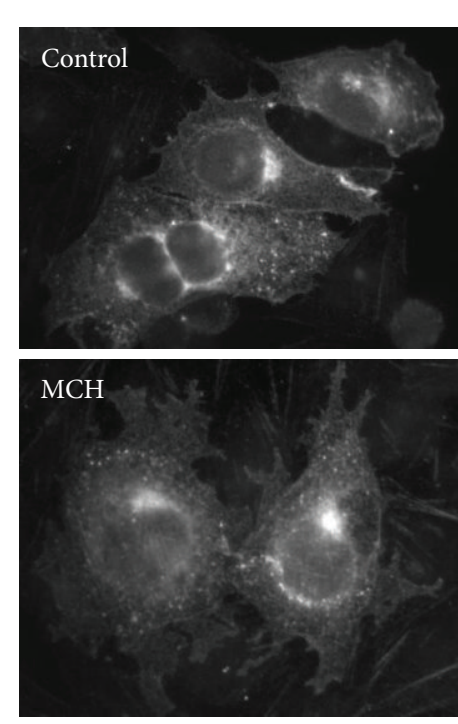

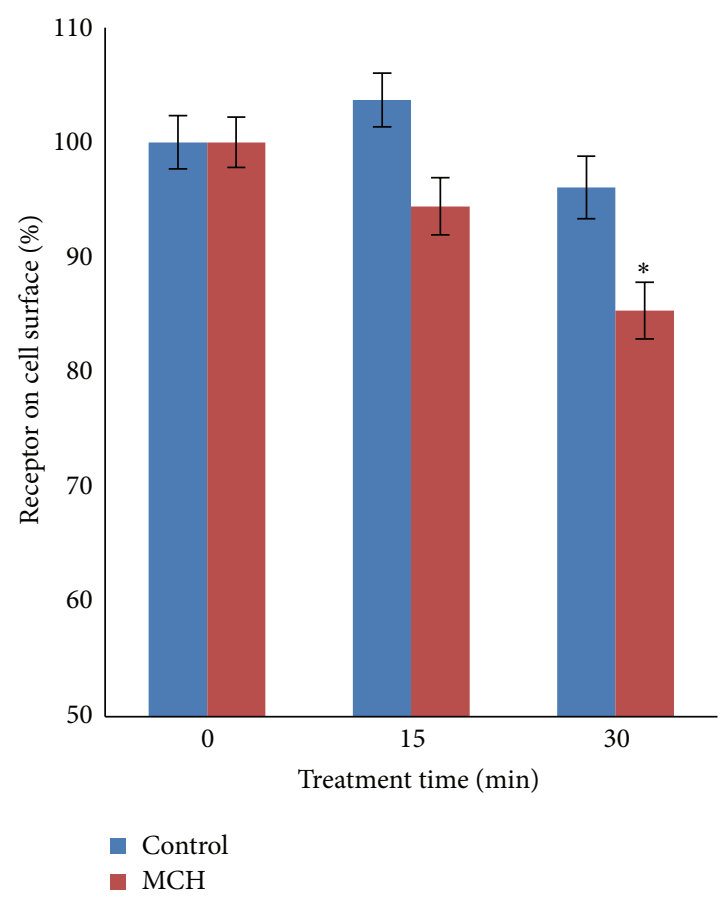

(b)

Figure 2: MCH initiates the removal of some MCHR1 from the plasma membrane. BHK-570 cells were transfected with VSVg-tagged MCHR1. (a) Untreated cells and cells that were exposed to $1 \mu \mathrm{M} \mathrm{MCH}$ for 30 min were fixed and immunostained with mouse anti-VSVg primary antibody and goat anti-mouse Alexa Fluor 488 secondary antibody. (b) The loss of MCHR1 from the plasma membrane in response to $1 \mu \mathrm{M} \mathrm{MCH}$ was measured using a modified cell-based ELISA protocol. Background signal from mock-transfected cells was subtracted. $n=18$ (6 triplicate experiments). * denotes statistical significance at $P<0.0025$ when compared to $100 \%$ control using Student's $t$-test.

was not sensitive enough to capture the receptors being internalized so we decided to utilize a modified cell-surface ELISA [14] to measure agonist-mediated internalization of an N-terminal VSVg-tagged MCHR1. The VSVg-tag allowed us to utilize a high-affinity antibody for this assay. We measured surface-localized receptor in untreated cells $(100 \% \pm 2.3 \%)$, and compared to cells treated with $\mathrm{MCH}$ for 15 or $30 \mathrm{~min}$, the results of which are shown in Figure 2(b). It was quite surprising that $\mathrm{MCH}$ was only able to drive surface receptor levels after $15 \mathrm{~min}$ to $96 \% \pm 2.7 \%$ of control and after $30 \mathrm{~min}$ to $85 \% \pm 2.5 \%(P<0.0025)$ of control, which was confirmed by fluorescence microscopy (Figure 2(a)). To determine if high expression levels of VSVg-MCHR1 contributed to the low internalization levels, we titrated back plasmid DNA amounts during transfection and found that it did not improve internalization (data not shown). This low level of internalization was also not a consequence of the $\mathrm{N}$ terminal tag because Murdoch and colleagues were able to observe internalization of this construct in HEK 293 cells by fluorescence microscopy [15]. Others utilized flow cytometry to measure $\mathrm{MCH}$-mediated removal of MCHR1 from the plasma membrane $[10,11]$.

3.3. Overexpression of $\beta$-Arrestins Rescues $\mathrm{MCH}$-Mediated MCHR1 Internalization. We wondered whether MCHR1 could interact with the machinery to internalize in our model system, and so we decided to coexpress VSVg-MCHR1 with either $\beta$-arrestin 1 or $\beta$-arrestin 2 and again measure internalization. Overexpression of $\beta$-arrestins has been shown to increase the internalization of $\beta_{2}$-adrenergic receptors [16] and it was hypothesized that $\beta$-arrestin would increase the rate of MCHR1 internalization in response to $\mathrm{MCH}$. When $\beta$-arrestin 1 was overexpressed, surface-localized MCHR1 unexpectedly declined in the absence of agonist from $100 \% \pm$ $3.4 \%$ to $92 \% \pm 2.6 \%$ at $15 \mathrm{~min}$ and $79 \% \pm 2.2 \%(P<0.01)$ at $30 \mathrm{~min}$. Treatment with $\mathrm{MCH}$ however further improved the removal of MCHR1 from the cell surface to $88 \% \pm$ $2.9 \%$ of the total at $15 \mathrm{~min}$ and only $64 \% \pm 2.4 \%$ after 30 minutes (Figure 3(a)). When this experiment was repeated with $\beta$-arrestin 2 , in contrast to $\beta$-arrestin 1 we found steady surface levels of MCHR1 over the course of $30 \mathrm{~min}$ in the absence of agonist. When cells were treated with hormone for $15 \mathrm{~min}$, surface receptor levels fell from $100 \% \pm 6.5 \%$ to $83 \% \pm 4.6 \%$ and by $30 \mathrm{~min}$ declined to $62 \% \pm 4.0 \%$ $(P<0.01)$ (Figure 3(b)). We directly compared the effects that each $\beta$-arrestin has on loss of MCHR1 from the cell surface of BHK-570 cells treated with $\mathrm{MCH}$ for $30 \mathrm{~min}$ in Figure 4. In our most successful internalization experiments, $60 \%$ of the receptor still remained on the plasma membrane despite vast overexpression of arrestins (data not shown) and supraphysiological agonist concentrations. Coexpression of MCHR1 with $\beta$-arrestin 1 resulted in an agonist-independent 


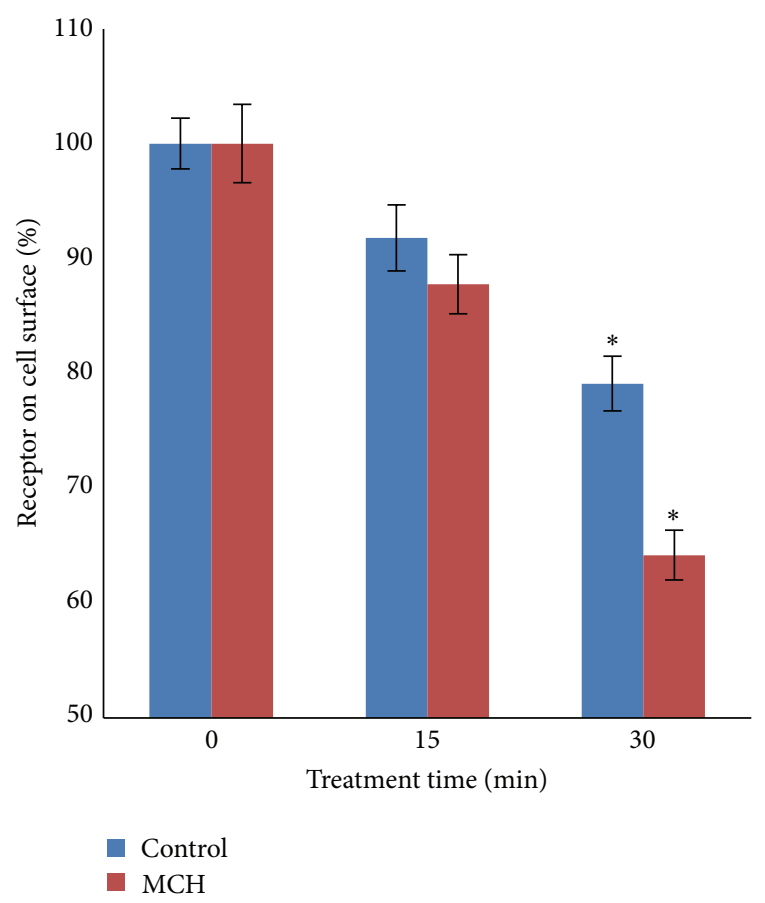

(a)

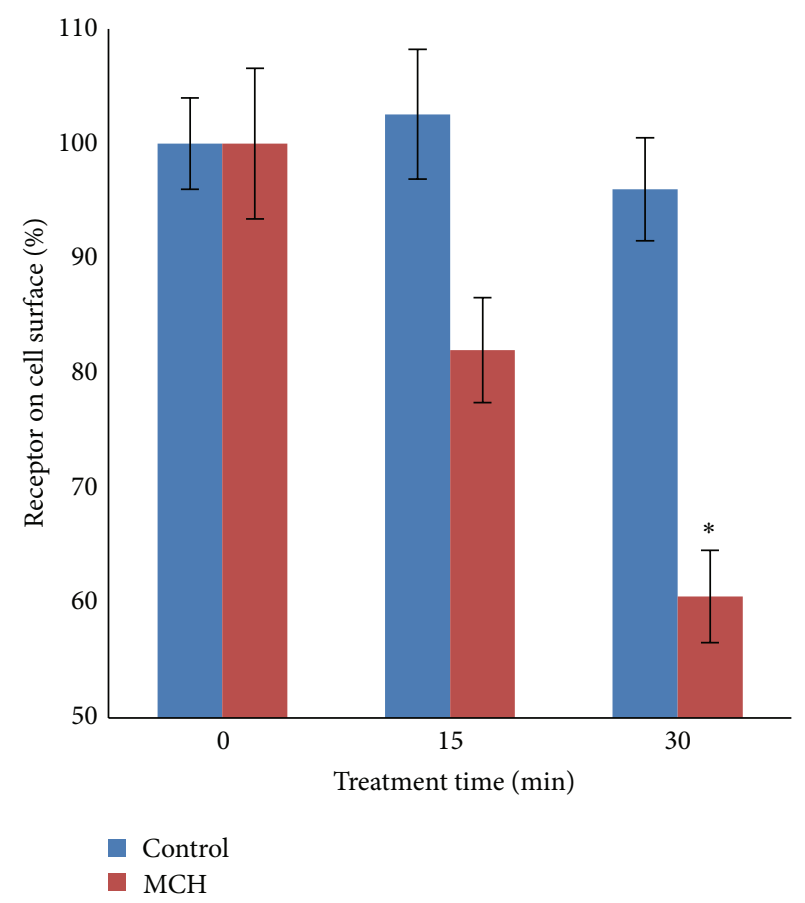

(b)

FIGURE 3: Coexpression of MCHR1 with $\beta$-arrestin 1 or 2 enhances MCH-mediated receptor internalization. BHK cells were cotransfected with $1 \mu \mathrm{g}$ each plasmids encoding VSVg-MCHR1 and (a) GFP $\beta$-arrestin 1 or (b) GFP $\beta$-arrestin 2 or GFP control plasmid (both (a) and (b)). The loss of MCHR1 from the plasma membrane in response to $1 \mu \mathrm{M} \mathrm{MCH}$ was measured using a modified cell-based ELISA protocol. Background signal from mock-transfected cells was subtracted. For $\beta$-arrestin $1, n=21$ ( 7 triplicate experiments); for $\beta$-arrestin $2, n=15$ ( 5 triplicate experiments). Ave \pm SEM plotted. ${ }^{*} P<0.01$.

net loss of $17.0 \%$ of MCHR1 from the plasma membrane and an agonist-dependent net loss of $21.1 \%$. The coexpression of $\beta$-arrestin 2 resulted in no appreciable agonist-independent net loss and an agonist-dependent net loss of $24.7 \%(P<$ $0.01)$.

These experiments highlight some differences between the association amongst the receptor and each arrestin; while both arrestins facilitated coupling of MCHR1 with the internalization machinery, $\beta$-arrestin 1 seems to have facilitated receptor coupling in the absence of agonist. To verify this visually, fluorescence microscopy was performed on fixed cells. $\beta$-arrestin 1 overexpression seems to result in a large amount of both receptor and arrestin to be stuck in a juxtanuclear compartment (Figure 4(b)). This could either be the result of inefficient trafficking of newly synthesized receptor to the plasma membrane or excessive removal of unoccupied receptors from the plasma membrane. Our ELISA data supports the latter hypothesis (Figures 3 and 4). Although some $\beta$-arrestin 1 was not visibly recruited to the plasma membrane in these cells (Figure 4(b), middle panel), treatment of cells with $\mathrm{MCH}$ for $30 \mathrm{~min}$ did show MCHR1 accumulation in punctate vesicles (Figure 4(b), top panel); these vesicles lacked $\beta$-arrestin 1 (Figure 4 (b), bottom panel). $\beta$-arrestin 2 gave a similar, but more extensive internalization profile with a larger number of MCHR1-positive puncta (Figure $4(\mathrm{c})$, top panel) that are also positive for GFP- $\beta$-arrestin 2 when the overlay is examined (Figure 4(c) middle and bottom panels). This data agrees with Evans and colleagues by demonstrating that the MCHR1- $\beta$-arrestin 2 interaction is of higher affinity for the receptor than that of $\beta$ arrestin 1 [10]. To further explore the potential colocalization of VSVg-MCHR1 with both $\beta$-arrestins, confocal microscopy was performed. Shown in Figure 5 is a representative single plane where colocalization of neither $\beta$-arrestin with VSVg-MCHR1 is readily apparent after a $30 \mathrm{~min}$ hormone treatment. Instead, most MCH receptor-containing vesicles are distinctly separate from $\beta$-arrestin-associated vesicles. Thus the apparent colocalization observed in Figure 4 could not be verified. Future experiments should explore this biochemically.

3.4. GRK2 Plays a Role in MCHR1 Internalization. G proteincoupled receptor kinase 2 (GRK2) is a family member of a group of protein kinases that generally function to regulate GPCR signaling through the phosphorylation of serine and threonine residues located on the intracellular loops and C-terminal tails of GPCRs. The addition of phosphate groups to these areas has been shown to terminate signaling by inhibiting $\mathrm{G}$ protein coupling and facilitating receptor internalization by increasing the affinity of arrestins for the receptor [17]. GRK2 plays a role in both $\beta$-arrestindependent and $\beta$-arrestin-independent trafficking of GPCRs [18]. A dominant-negative form of GRK2, GRK2 K220R has 


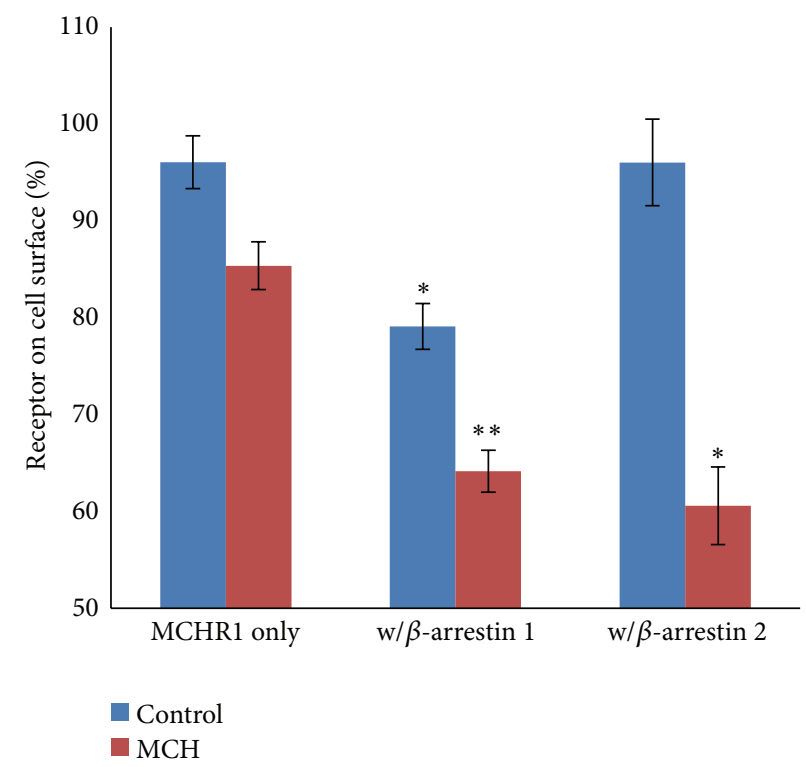

(a)

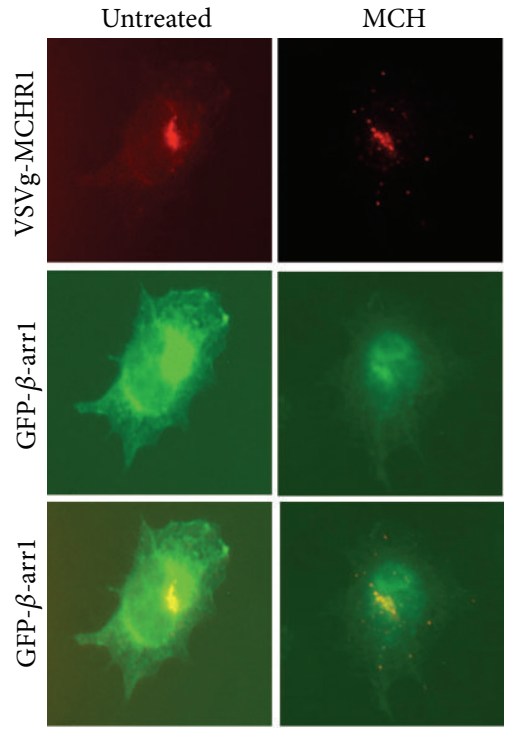

(b)

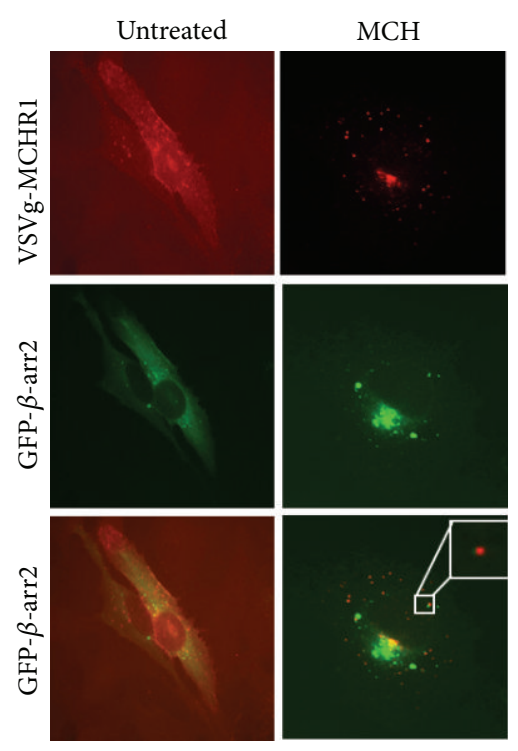

(c)

FIGURE 4: Overexpression of $\beta$-arrestins differentially influences MCHR1 internalization. BHK-570 cells were transfected with VSVg-MCHR1 and either $\beta$ arrestin-1 or $\beta$ arrestin-2. (a) Cells were treated with $1 \mu \mathrm{M} \mathrm{MCH}$ or vehicle for 30 min and internalization was measured utilizing a cell-based ELISA. Data from Figure 3 was replotted. Ave \pm SEM plotted. ${ }^{*}{ }^{*} P<0.005,{ }^{*} P<0.01$. VSVg-MCHR1 and GFP $\beta$-arrestin 1-expressing cells (b) and VSVg-MCHR1 and GFP $\beta$-arrestin 2-expressing cells (c) were treated with $1 \mu \mathrm{M}$ MCH or vehicle for 30 min, then fixed, and immunostained with anti-VSVg antibody. Fluorescence microscopy was performed and overlay images compared.

already been characterized [19] and is known to knock down the expression of wild-type GRK2 when coexpressed. Based on previous studies [20], it was hypothesized that the overexpression of GRK2 would increase the rate of internalization, while transfection of the K220R GRK2 would knock down endogenous GRK2 resulting in inhibited MCHR1 internalization. MCHR1 internalization was monitored by cell-surface ELISA over the course of $30 \mathrm{~min}$. Cotransfection of MCHR1 with either GRK2 or K220R GRK2 resulted in no appreciable loss of receptors in the absence of agonist (Figure 6). An agonist-dependent loss of receptors equating to $6.5 \%(P<$ $0.005)$ for GRK2-expressing cells was measured and a net increase in surface receptor levels of $11.3 \%(P<0.005)$ for K220R-expressing cells was measured when compared to cells given a control plasmid.

3.5. MCH Signaling to a Leptin Promoter Is Attenuated by Coexpression of Arrestins. In order to determine if this loss of 


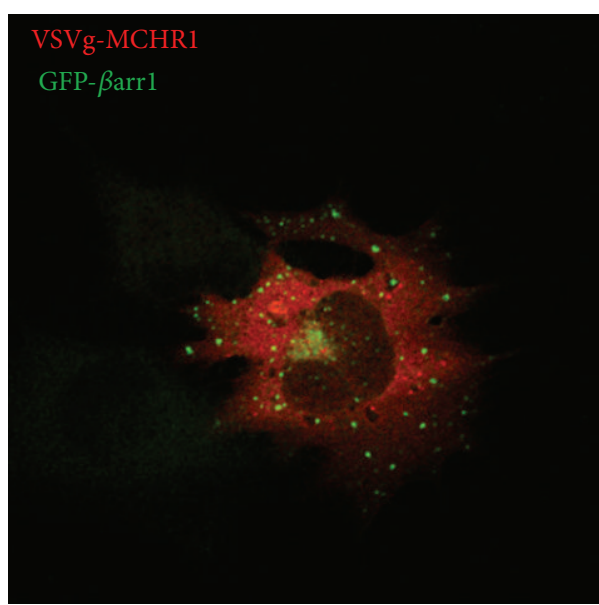

(a)

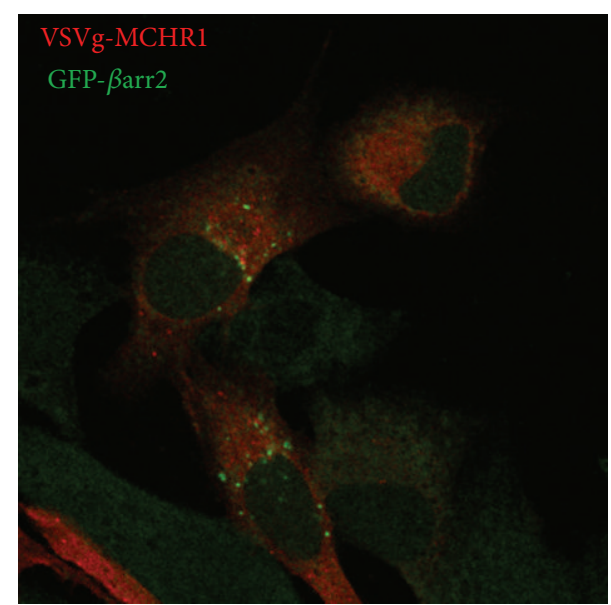

(b)

FIGURE 5: VSVg-MCHR1-containing vesicles are distinct from those associated with $\beta$-arrestins. VSVg-MCHR1 and GFP $\beta$-arrestin 1expressing cells (a) and VSVg-MCHR1 and GFP $\beta$-arrestin 2-expressing cells (b) were treated with $1 \mu \mathrm{M}$ MCH for 30 min, then fixed, and immunostained with anti-VSVg antibody. Confocal microscopy was performed using a grid pattern confocal microscope and Image-Pro Plus software. A single slice is shown as an overlay of both Alexa Fluor $(546 \mathrm{~nm})$ and GFP $(488 \mathrm{~nm})$ signals.

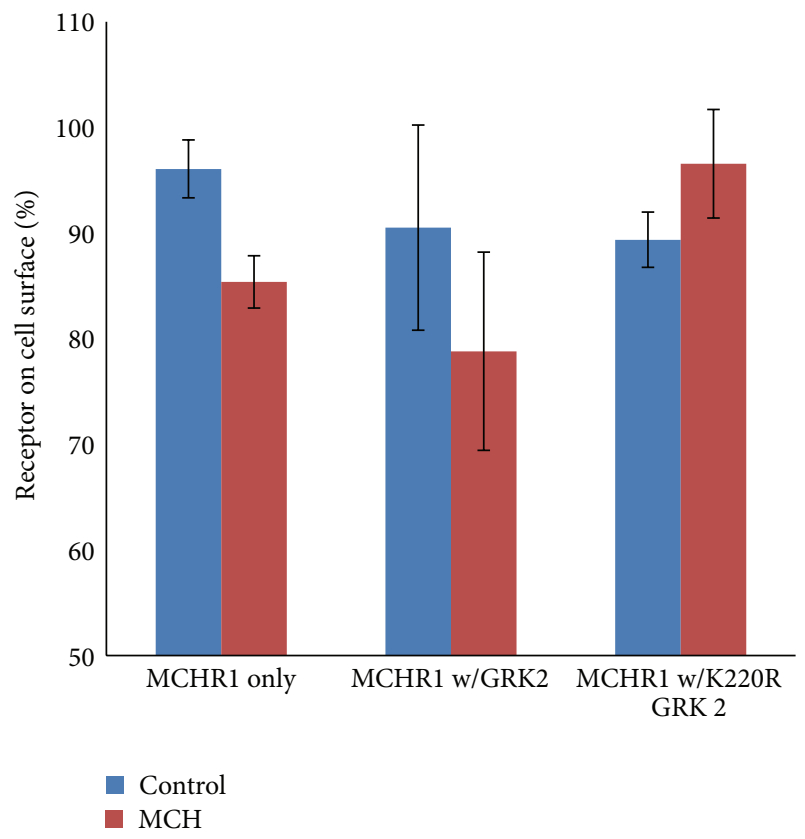

FIGURE 6: Perturbations of cellular GRK2 levels affect MCHmediated internalization of MCHR1. BHK570 cells were transfected with VSVg-MCHR1 and either GRK2 or K220R GRK2. Cells were treated with $1 \mu \mathrm{M} \mathrm{MCH}$ or vehicle for 30 min and internalization was measured utilizing a cell-based ELISA. $n=18$ for control; $n=15$ for GRK2; $n=9$ for K220R GRK2. Ave \pm SEM plotted. Experiment reached the $90 \%$ confidence interval but did not reach statistical significance.

receptor from the cell surface translates into improved desensitization of $\mathrm{MCH}$ signaling, we utilized a reporter plasmid from which luciferase expression is driven by activation of the leptin promoter. This construct was previously reported

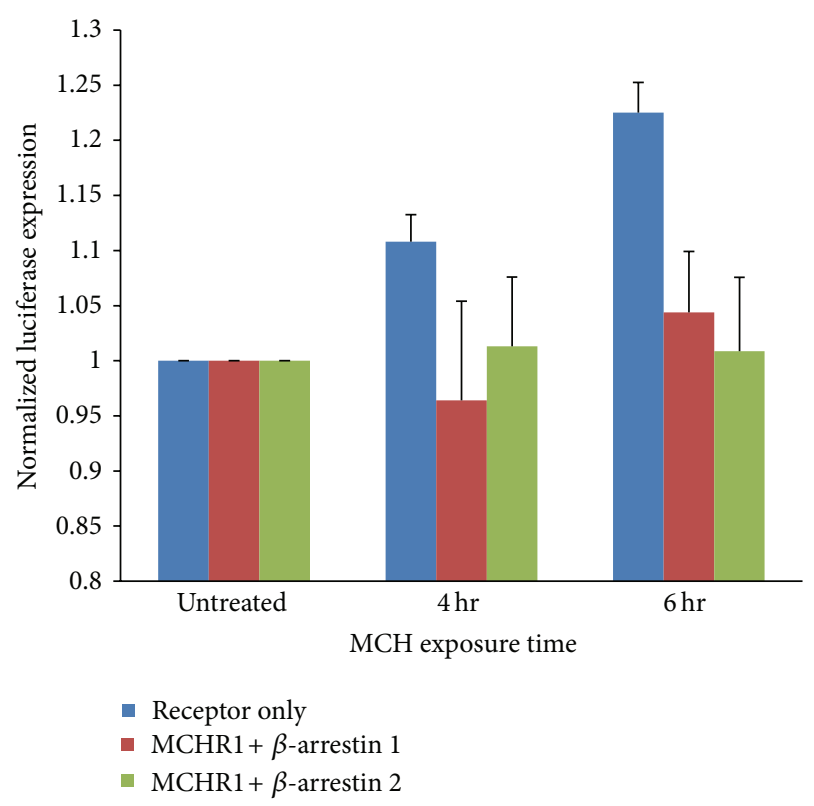

FIGURE 7: $\beta$-arrestins diminish the activation of a leptin promoter by MCH. BHK 570 cells triple transfected with either (1) MCHR1/p(762)ob luc/GFP in pcDNA3, (2) MCHR1/p(-762)ob luc/GFP- $\beta$ arrestin 1, or (3) MCHR1/p(-762)ob luc/GFP- $\beta$-arrestin 2 were treated with $1 \mu \mathrm{M} \mathrm{MCH}$ for up to $6 \mathrm{~h}$. A luciferase assay was then performed to quantifiably measure the activation of the leptin promoter. $n=5$ for control and $n=3$ for each $\beta$-arrestin experiment. Graph shows averages of normalized data \pm SEM. Experiment reached the $85 \%(\beta$-arr2) and 90\% ( $\beta$-arr1) confidence intervals but did not reach statistical significance.

to respond to $\mathrm{MCH}$ over the course of several hours [21]. As shown in Figure 7, cells expressing $\beta$-arrestin 1 or 2 showed less luciferase expression compared to control in 
response to $\mathrm{MCH}$ treatment for up to $6 \mathrm{~h}$. This indicates that $\mathrm{MCH}$-mediated activation of the leptin promoter can be desensitized by overexpression of arrestins and it supports the hypothesis that agonist-mediated internalization of MCHR1 acts to desensitize cells to $\mathrm{MCH}$.

\section{Discussion}

Melanin-concentrating hormone acts on at least two G protein-coupled receptors in the central nervous system and peripheral organs to elucidate a variety of physiological responses. A few early studies suggested that at least one of those receptors, melanin-concentrating hormone receptor 1 , undergoes phosphorylation, $\beta$-arrestin 2 recruitment, and agonist-mediated internalization [9-11]. However, when we attempted to replicate these experiments much to our surprise we were unable to measure internalization of MCHR1 visually (Figure 2(a)) and when measured quantitatively, only weak $(\sim 15 \%)$ internalization was observed (Figures $2(\mathrm{~b})$ and 3) despite extensive desensitization of the ERK pathway (Figure 1). Our observations were not limited to BHK-570 cells, but MCHR1 internalization was also not observed in $\mathrm{CHO}-\mathrm{K} 1$ cells or 3T3-L1 cells for various receptor constructs including MCHR1-eYFP (data not shown). This is in contrast to results described by others who observed internalization of MCHR1 by other means [9-11]. The clathrin-mediated endocytosis pathway has been widely characterized from studies of other GPCRs [22]. $\beta$-arrestins are important mediators, helping to recruit clathrin heavy chain to GPCRs, and receptors such as the $\beta_{2}$-adrenergic receptor are known to desensitize through this pathway [21-23].

The relative levels of GPCR, $\beta$-arrestin, and GRK have been previously implicated in sequestration efficiency as reported by Barak and colleagues for the $\beta_{2}$-adrenergic receptor [24]; however, these differences probably do not entirely explain the results we see with MCHR1. mGlu1R $\beta$ [25] and GHRH-R [26] are two GPCRs that when heterologously expressed in BHK-570 cells internalized rapidly following agonist exposure, suggesting that baby hamster kidney fibroblast cells indeed have the capacity to sequester GPCRs. When we coexpressed $\beta$-arrestins 1 and 2 or GRK2 with MCHR1, we were able to partially rescue internalization of the receptor (Figures 3-4). Our results suggest that $\beta$-arrestin 1 is indeed capable of coupling MCHR1 to the endocytic machinery, but that the association is weak because cointernalization of the arrestin with the receptor was not observed (Figure 4(b)). We also presented evidence of agonist-independent removal of MCHR1 from the cell surface with $\beta$-arrestin 1 in Figures 3(a) and 4(a), although this could be the result of an antibody-induced conformational change that results in internalization of the receptor. When $\beta$ arrestin 2 was coexpressed, co-internalization with MCHR1 following agonist treatment was observed (Figure 4(c)), but not confirmed with confocal microscopy methods (Figure 5). These results mirror those from a study done with $\beta_{2}$ adrenergic receptor in which cells triple transfected with $\beta_{2}$ adrenergic receptor, $\beta_{2}$-adrenergic receptor kinase, and $\beta$ arrestins showed a significant synergistic effect [24].
The suggestion that MCHR1 favors $\beta$-arrestin 2 is not a new one. Evans et al. previously reported selective, but transient recruitment of $\beta$-arrestin 2 to the plasma membrane of transfected HEK293 cells following MCH exposure [10]. Unlike their study, in our experimental system utilizing BHK570 cells it was not necessary to overexpress GRK2 to observe either $\beta$-arrestins' effect on MCHR1 internalization. Also, although we did not specifically detect recruitment of the GFP $\beta$-arrestins to the cell surface, it is implied since their presence efficiently facilitated coalescing of receptors into punctate vesicles for internalization. Saito and colleagues, using HEK293T cells, overexpressed $\beta$-arrestin 2 and showed no significant effects on $\mathrm{MCH}$-mediated receptor internalization. However, a dominant-negative version seemed to inhibit it [11]. These seemingly conflicting results may have been the result of inefficient GRK2 levels to accompany the overexpressed $\beta$-arrestin 2. The best illustration of cellto-cell variation in internalization efficiency is for the $\beta_{2}$ adrenergic receptor [24], and abnormalities in GRK or $\beta$ arrestin protein levels have been linked to Alzheimer's disease [27], inflammatory diseases [28], and cardiac ailments [17]. Our results illustrate that protein levels of both GRK2 and $\beta$ arrestin have the potential to modulate the response of cells to $\mathrm{MCH}$, potentially influencing human appetite.

$G$ protein-coupled receptors can be divided into two classes: Class A receptors and Class B receptors. Class A receptors preferentially bind $\beta$-arrestin 2 over $\beta$-arrestin 1 and their interaction is transient in nature with $\beta$-arrestin and the receptor dissociating prior to entering the endosome. Class $\mathrm{B}$ receptors have equal affinity for both $\beta$-arrestin 1 and $\beta$-arrestin 2 and form stable interactions resulting in the translocation of both receptor and $\beta$-arrestin into the endosome [29]. When both $\beta$-arrestins were coexpressed with MCHR1 in this study, receptor internalization was facilitated significantly over control, but to a greater extent with $\beta$-arrestin 2, suggesting that MCHR1 is a Class A receptor. If cointernalization of $\beta$-arrestin 2 with MCHR1 really does occur in our model system, it would suggest that MCHR1 is a Class B receptor. MCHR1 may have an intermediate phenotype in BHK-570 cells.

The protein kinase GRK2 is known to phosphorylate serine threonine residues on the third intracellular loop and C-terminal tail of GPCRs increasing their affinity for $\beta$ arrestins in an agonist-dependant manner [17]. Up until now, the effect of GRK2 overexpression on the desensitization of MCHR1 had yet to be explicitly measured although, as already stated, Evans et al. utilized GRK2 transfected cells while observing the nature of the interaction of $\beta$ arrestins with MCHR1 [10]. Agonist-induced internalization of MCHR1 while overexpressing dominant-negative GRK 2 showed no significant increase in the rate of receptor internalization (Figure 5) similar to the $\mu$-opioid receptors. These receptors are dependent on phosphorylation by GRKs for the recruitment of $\beta$-arrestins in contrast to that of the $\beta_{2}$ adrenergic receptor, which can interact with $\beta$-arrestins in the absence of GRK2 phosphorylation sites [23, 24]. This suggests that MCHR1 is dependent on GRK2 phosphorylation for the recruitment of $\beta$-arrestins and together with previous data and that the rate-limiting step in MCHR1 desensitization is 
dependent on the cellular concentration of $\beta$-arrestins and/or GRK2. This would be true, however, if internalization was required for desensitization of $\mathrm{MCH}$ signaling. Considering that only $15 \%$ of receptors internalized in our model system (Figure 2) yet ERK signaling was efficiently desensitized (Figure 1), we hypothesize that desensitization of the $\mathrm{MCH}$ signaling pathway in these cells does not solely rely on removal of receptors from the plasma membrane.

We hypothesized that overexpression of arrestins would not only promote agonist-mediated internalization of MCHR1, but that this would indeed translate into a desensitization of downstream signaling. Our results in Figure 7 support a role for $\beta$-arrestins in downregulating this pathway because $\mathrm{MCH}$-mediated luciferase accumulation is essentially absent when they are coexpressed with MCHR1. This suggests that $\mathrm{MCH}$-mediated ERK signaling is spatially linked to membrane-localized MCHR1 and that agonistmediated internalization of MCHR1 is not necessary for ERK signaling, rather it may participate in signal termination.

MCHR1 signaling to $G$ proteins is thought to be regulated differentially by at least two RGS proteins, RGS2 and RGS8 $[25,26]$. These proteins act to promote GTP hydrolysis inactivating the $\mathrm{G}$ protein signal. MCH-mediated ERK signaling in HEK293 cells proceeds via $\mathrm{G} \alpha \mathrm{q}$ and $\mathrm{G} \alpha \mathrm{i}$ [30], both of which are targets of RGS8 $[25,26]$. It is not known whether RGS8 plays a role in desensitizing ERK signaling in BHK-570 cells, but it seems a likely candidate.

Another likely possibility is that MCHR1 becomes spatially segregated from its signaling components in the plasma membrane. MCHR1 forms a complex with caveolin-1 in these cells, and MCHR1 is highly enriched in caveolae [13]. It has been reported that $\mathrm{G} \alpha \mathrm{i}$ and $\mathrm{G} \alpha \mathrm{s}$ are able to migrate into and out of caveolae unlike G $\alpha$ q, which is tethered there [31]. Interestingly, adipocytes express exceedingly high levels of caveolin-1; therefore regulation of $\mathrm{MCH}$ signaling via caveolae or other lipid rafts in this cell type seems to be a strong possibility. The obese phenotype seen as a result of primary cilia loss in Bardet-Biedl syndrome is hypothesized to be the result of a loss of cilia-localized MCHR1 in the brain [7]. Since $\mathrm{MCH}$ signals appetite, this suggests that ciliary localization of MCHR1 dampens the MCH signal, further evidence to suggest that spatial organization of these receptors in the plasma membrane contributes to the regulation of its activity.

\section{Conclusion}

ERK signaling by $\mathrm{MCH}$ potently desensitizes in BHK-570 cells. We tested the hypothesis that agonist-induced removal of $\mathrm{MCH}$ receptors from the plasma membrane was largely responsible for this process. Surprisingly we found that $\mathrm{MCH}$ receptors internalize very poorly in BHK-570 cells unless $\beta$ arrestin 1 or $\beta$-arrestin 2 is overexpressed. Similarly, GRK2 phosphorylation of MCHR1 is thought to be important because a dominant-negative GRK2 was able to eliminate even the small amount of receptor internalization induced by $\mathrm{MCH}$. We conclude that $\mathrm{MCH}$ receptor signaling and desensitization are particularly sensitive to cellular levels of $\beta$-arrestins and GRK2, which should be strongly taken under consideration when interpreting $\mathrm{MCH}$ signaling studies across different cell types. Perturbation of the cellular levels of these accessory proteins could greatly influence the activity of this appetite-stimulating pathway.

\section{Conflict of Interests}

The author(s) declare(s) that there is no conflict of interests regarding the publication of this paper.

\section{Acknowledgment}

This work was supported by a grant from the National Institutes of Health (GM090163) to L. B. Cook.

\section{References}

[1] H. Kawauchi, I. Kawazoe, and M. Tsubokawa, "Characterization of melaninconcentrating hormone in chum salmon pituitaries," Nature, vol. 305, no. 5932, pp. 321-323, 1983.

[2] P. Pissios and E. Maratos-Flier, "Melanin-concentrating hormone: from fish skin to skinny mammals," Trends in Endocrinology and Metabolism, vol. 14, no. 5, pp. 243-248, 2003.

[3] D. Qu, D. S. Ludwig, S. Gammeltoft et al., "A role for melaninconcentrating hormone in the central regulation of feeding behaviour," Nature, vol. 380, no. 6571, pp. 243-247, 1996.

[4] D. S. Ludwig, N. A. Tritos, J. W. Mastaitis et al., "Melaninconcentrating hormone overexpression in transgenic mice leads to obesity and insulin resistance," The Journal of Clinical Investigation, vol. 107, no. 3, pp. 379-386, 2001.

[5] M. Shimada, N. A. Tritos, B. B. Lowell, J. S. Flier, and E. Maratos-Flier, "Mice lacking melanin-concentrating hormone are hypophagic and lean," Nature, vol. 396, no. 6712, pp. 670674, 1998.

[6] Y. Chen, C. Hu, C. Hsu et al., "Targeted disruption of the melanin-concentrating hormone receptor-1 results in hyperphagia and resistance to diet-induced obesity," Endocrinology, vol. 143, no. 7, pp. 2469-2477, 2002.

[7] N. F. Berbari, J. S. Lewis, G. A. Bishop, C. C. Askwith, and K. Mykytyn, "Bardet-Biedl syndrome proteins are required for the localization of G protein-coupled receptors to primary cilia," Proceedings of the National Academy of Sciences of the United States of America, vol. 105, no. 11, pp. 4242-4246, 2008.

[8] Y. Meleco, "AMRI Announces Successful Completion of Phase I Clinical Study of Obesity Compound," Business Wire, May 2011, http://www.businesswire.com/news/home/20110531005247/en/ AMRI-Announces-Successful-Completion-Phase-Clinical-Study.

[9] P. M. C. Lembo, E. Grazzini, J. Cao et al., “The receptor for the orexigenic peptide melanin-concentrating hormone is a Gprotein-coupled receptor," Nature Cell Biology, vol. 1, no. 5, pp. 267-271, 1999.

[10] N. A. Evans, D. A. Groarke, J. Warrack et al., "Visualizing differences in ligand-induced $\beta$-arrestin-GFP interactions and trafficking between three recently characterized G proteincoupled receptors," Journal of Neurochemistry, vol. 77, no. 2, pp. 476-485, 2001.

[11] Y. Saito, M. Tetsuka, Y. Li, H. Kurose, and K. Maruyama, "Properties of rat melanin-concentrating hormone receptor 1 internalization," Peptides, vol. 25, no. 10, pp. 1597-1604, 2004. 
[12] R. L. Bradley, J. P. R. Mansfield, E. Maratos-Flier, and B. Cheatham, "Melanin-concentrating hormone activates signaling pathways in 3T3-L1 adipocytes," American Journal of Physiology-Endocrinology and Metabolism, vol. 283, no. 3, pp. E584-E592, 2002.

[13] L. B. Cook, E. B. Delorme-Axford, and K. Robinson, "Caveolae as potential mediators of MCH-signaling pathways," Biochemical and Biophysical Research Communications, vol. 375, no. 4, pp. 592-595, 2008.

[14] B. W. Jones, J. S. Gyun, E. K. Greuber, and P. M. Hinkle, "Phosphorylation of the endogenous thyrotropin-releasing hormone receptor in pituitary $\mathrm{GH} 3$ cells and pituitary tissue revealed by phosphosite-specific antibodies," The Journal of Biological Chemistry, vol. 282, no. 17, pp. 12893-12906, 2007.

[15] H. Murdoch, G. Feng, D. Bächner et al., "Periplakin interferes with $G$ protein activation by the melanin- concentrating hormone receptor-1 by binding to the proximal segment of the receptor C-terminal tail," The Journal of Biological Chemistry, vol. 280, no. 9, pp. 8208-8220, 2005.

[16] S. Pippig, S. Andexinger, K. Daniel et al., "Overexpression of $\beta$-arrestin and $\beta$-adrenergic receptor kinase augment desensitization of $\beta 2$-adrenergic receptors," The Journal of Biological Chemistry, vol. 268, no. 5, pp. 3201-3208, 1993.

[17] R. T. Premont and R. R. Gainetdinov, "Physiological roles of G protein-coupled receptor kinases and arrestins," Annual Review of Physiology, vol. 69, pp. 511-534, 2007.

[18] T. Evron, T. L. Daigle, and M. G. Caron, "GRK2: multiple roles beyond G protein-coupled receptor desensitization," Trends in Pharmacological Sciences, vol. 33, no. 3, pp. 154-164, 2012.

[19] G. Kong, R. Penn, and J. L. Benovic, "A $\beta$-adrenergic receptor kinase dominant negative mutant attenuates desensitization of the $\beta 2$-adrenergic receptor," The Journal of Biological Chemistry, vol. 269, no. 18, pp. 13084-13087, 1994.

[20] J. I. Moden, An Investigation of Melanin-Concentrating Hormone Receptor Internalization-Or a Lack Thereof, The College at Brockport, State University of New York, Brockport, NY, USA, 2012.

[21] Y. He, H. Chen, M. J. Quon, and M. Reitman, "The mouse obese gene. Genomic organization, promoter activity, and activation by CCAAT/enhancer-binding protein $\alpha$," The Journal of Biological Chemistry, vol. 270, no. 48, pp. 28887-28891, 1995.

[22] C. Le Roy and J. L. Wrana, "Clathrin- and non-clathrinmediated endocytic regulation of cell signalling," Nature Reviews Molecular Cell Biology, vol. 6, no. 2, pp. 112-126, 2005.

[23] M. J. Lohse, S. Andexinger, J. Pitcher et al., "Receptor-specific desensitization with purified proteins. Kinase dependence and receptor specificity of $\beta$-arrestin and arrestin in the $\beta 2$ - adrenergic receptor and rhodopsin systems," The Journal of Biological Chemistry, vol. 267, no. 12, pp. 8558-8564, 1992.

[24] L. Ménard, S. S. G. Ferguson, J. Zhang et al., "Synergistic regulation of $\beta 2$-adrenergic receptor sequestration: Intracellular complement of $\beta$-adrenergic receptor kinase and $\beta$-arrestin determine kinetics of internalization," Molecular Pharmacology, vol. 51, no. 5, pp. 800-808, 1997.

[25] F. Ciruela and R. A. J. McIlhinney, "Differential internalisation of mGluR1 splice variants in response to agonist and phorbol esters in permanently transfected BHK cells," FEBS Letters, vol. 418, no. 1-2, pp. 83-86, 1997.

[26] C. Veyrat-Durebex, L. Pomerleau, D. Langlois, and P. Gaudreau, "Internalization and trafficking of the human and rat growth hormone-releasing hormone receptor," Journal of Cellular Physiology, vol. 203, no. 2, pp. 335-344, 2005.
[27] A. Thathiah, K. Horré, A. Snellinx et al., “ $\beta$-arrestin 2 regulates A $\beta$ generation and $\gamma$-secretase activity in Alzheimer's disease," Nature Medicine, vol. 19, no. 1, pp. 43-49, 2012.

[28] A. Vroon, C. J. Heijnen, and A. Kavelaars, "GRKs and arrestins: regulators of migration and inflammation," Journal of Leukocyte Biology, vol. 80, no. 6, pp. 1214-1221, 2006.

[29] S. K. Shenoy and R. J. Lefkowitz, "Multifaceted roles of $\beta$ arrestins in the regulation of seven-membrane-spanning receptor trafficking and signalling," Biochemical Journal, vol. 375, part 3, pp. 503-515, 2003.

[30] P. Pissios, D. J. Trombly, I. Tzameli, and E. Maratos-Flier, "Melanin-concentrating hormone receptor 1 activates extracellular signal-regulated kinase and synergizes with Gs-coupled pathways," Endocrinology, vol. 144, no. 8, pp. 3514-3523, 2003.

[31] P. Oh and J. E. Schnitzer, "Segregation of heterotrimeric G proteins in cell surface microdomains. $\mathrm{G}(\mathrm{q})$ binds caveolin to concentrate in caveolae, whereas $\mathrm{G}(\mathrm{i})$ and $\mathrm{G}(\mathrm{s})$ target lipid rafts by default," Molecular Biology of the Cell, vol. 12, no. 3, pp. 685698, 2001. 


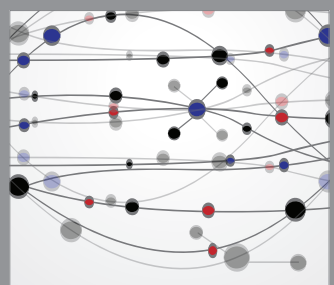

The Scientific World Journal
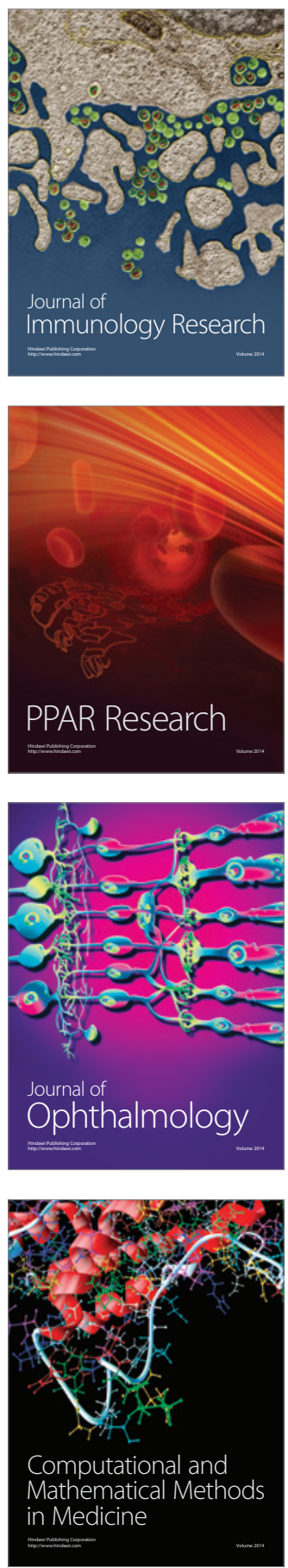

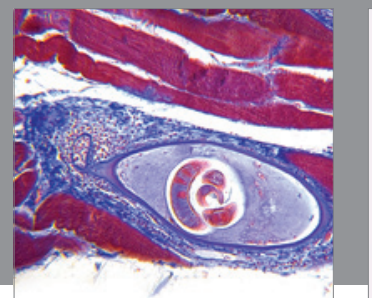

Gastroenterology

Research and Practice
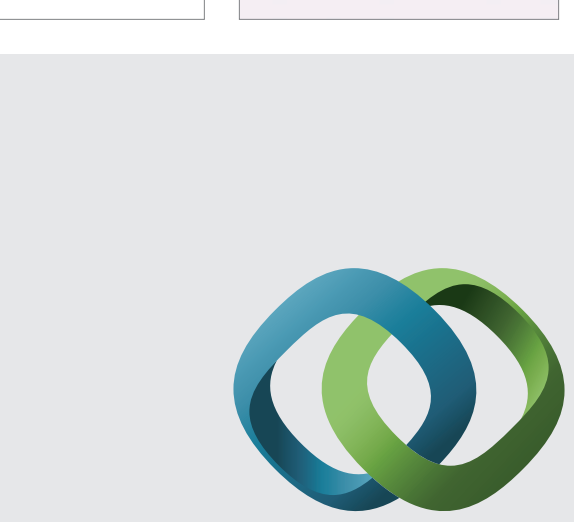

\section{Hindawi}

Submit your manuscripts at

http://www.hindawi.com
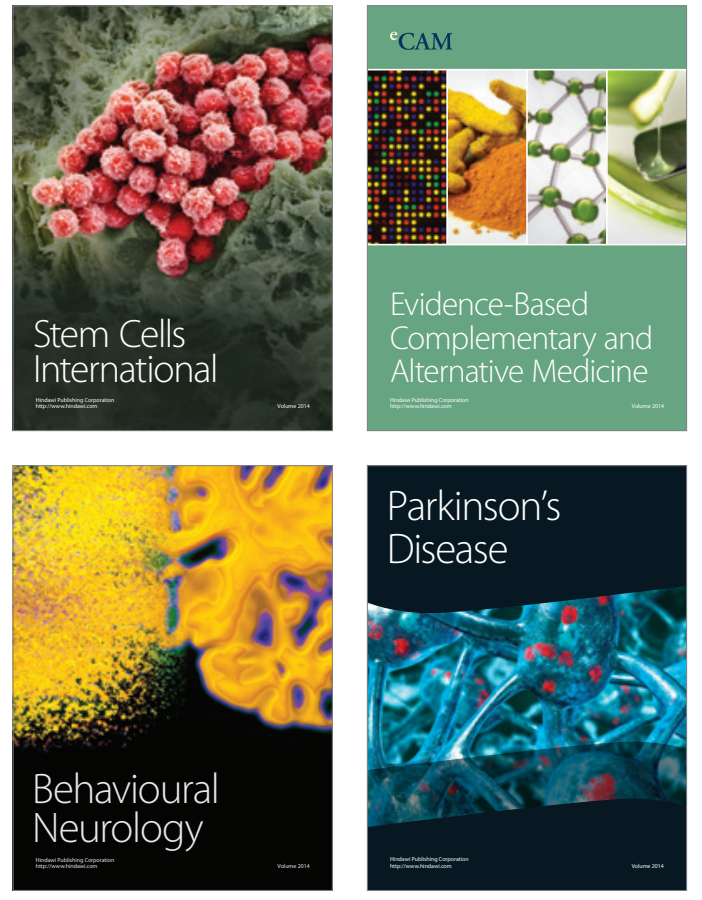
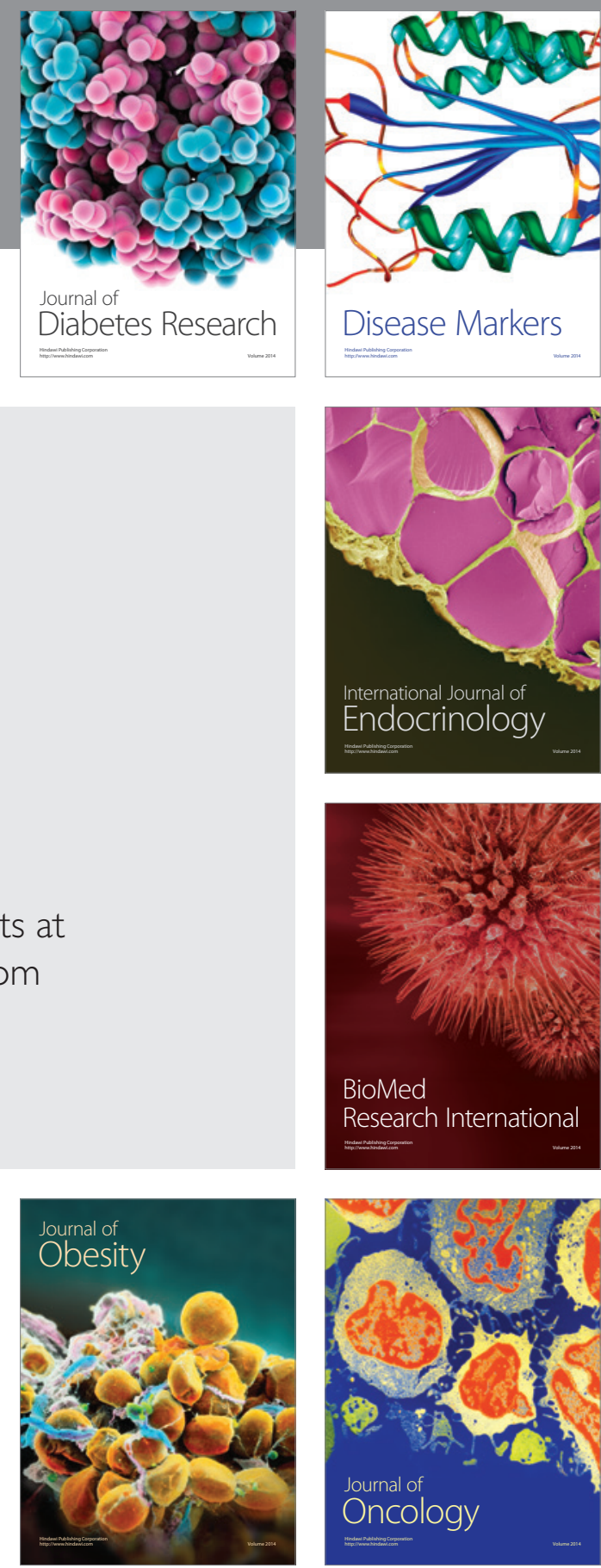

Disease Markers
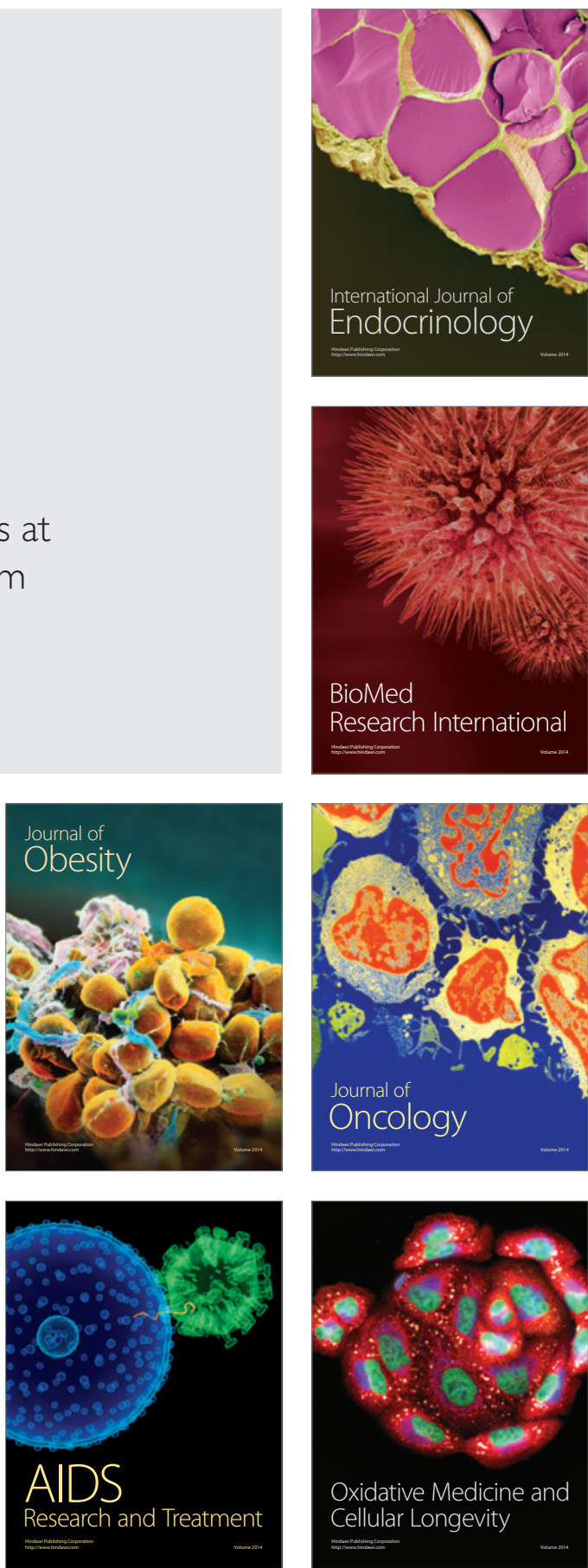